\title{
Quelques facteurs à prendre en considération pour réussir son cours en ligne
}

\author{
Some factors to take into consideration to success an on online \\ course
}

\section{Algunos factores a considerar para realizar con éxito un curso en línea}

RÉSUMÉ

\begin{abstract}
Les innovations numériques sont de plus en plus nombreuses dans le domaine de l'enseignement. Ces nouvelles pratiques nécessitent la prise en considération de certains paramètres pour un meilleur apprentissage. En effet, l'effet de la fascination technologique ou "la pseudo-autonomie" des apprenants peuvent inhiber l'apprentissage et constituer des points de blocage dans le processus de l'enseignement en ligne. Quant à la littératie numérique, la scénarisation des contenus, la proposition d'activités et de projets pour motiver les étudiants, ils peuvent fonder une nouvelle relation au savoir à l'ère du numérique. La pédagogie en présentiel diffère de celle qu'il faut adopter en ligne. Passer de l'espace métrique à l'espace virtuel impose des changements d'ordre pédagogique à ne pas négliger pour réussir un cours en ligne.
\end{abstract}

Mots-clés : enseignement numérique, fascination technologique, autonomie, scénarisation, pédagogie en ligne 
Digital innovations are increasing in the field of education. These new practices require the consideration of certain parameters for a better learning. Indeed the effect of technological fascination or "pseudo-autonomy" of learners can inhibit learning and constitute blocking points in the process of online education. As for digital literacy, scripting content, the proposal of activities and projects to motivate students, they can found a new relationship to knowledge in the digital age. Face-to-face pedagogy differs from that to be adopted online. Moving from the metric space to the virtual space imposes pedagogical changes that should not be neglected in order to pass an online course.

Keywords: digital education, technological fascination, autonomy, scriptwriting, online pedagogy

\section{RESUMEN}

Las innovaciones digitales están aumentando en el campo de la educación. Estas nuevas prácticas requieren la consideración de ciertos parámetros para un mejor aprendizaje. De hecho, el efecto de la "fascinación" o la "pseudo-autonomía" de los estudiantes puede inhibir el aprendizaje y constituir puntos de bloqueo en el proceso de educación en línea. En cuanto a la alfabetización digital, el contenido de scripts, la propuesta de actividades y proyectos para motivar a los estudiantes, pueden encontrar una nueva relación en la era digital. La pedagogía cara a cara difiere de la adoptada en línea. Pasar del espacio métrico al espacio virtual impone cambios pedagógicos que no deben descuidarse para pasar una carrera en línea.

Palabras clave: educación digital, fascinación tecnológica, autonomía, escritura de guiones, pedagogía en línea

\section{Introduction}

Le numérique a envahi notre vie quotidienne, notre milieu socio-professionnel, notre façon de nous informer, de vivre ou de nous distraire; il a même changé les relations humaines : de nos jours chacun d'entre nous est capable de créer une communauté ou d'en suivre une, de connaître instantanément le lieu où se trouve un ami, s'il est en ligne, le parcours de son voyage, ce qu'il visite, ce qu'il aime ou ce qu'il n'aime pas... En effet, la vraie nouveauté avec le numérique c'est d'avoir accéléré l'accès universel aux personnes et à l'information à travers les réseaux sociaux, les GPS et les moteurs de recherche. Sur la sphère de la googlisation et de l'ubérisation, la planète, l'humanité, le savoir sont à portée de main. Actuellement, nous avons le monde sur nos Smartphones!

C'est ainsi que le numérique a changé notre relation au savoir. Si le monde de la connaissance a connu deux grandes révolutions principales, celle du passage de l'oral à l'écrit et celle du passage de l'écrit à l'imprimé, le numérique est considéré comme la troisième révolution, caractérisée par un immense basculement comparable à celui de la fin de l'Empire romain ou de la renaissance. Nous assistons à la naissance d'une nouvelle culture participative où la création et le partage de contenus en ligne tendent à devenir une norme; une chose est sûre dans ce magma de l'information : notre rapport à la connaissance s'est complètement métamorphosé et il ne serait plus question de retour en arrière. Montaigne parlait déjà, 
suite à l'invention de l'imprimerie, d'une " tête bien faite plutôt que d'une tête bien pleine ", ca s'il était auparavant impératif de connaître par cœur l'intégralité du savoir, il suffisait, avec la propagation du livre, de savoir où trouver l'information. Actuellement, nous n'avons même pas besoin de savoir où chercher l'information puisqu'il suffit de saisir un mot sur un moteur de recherche pour trouver tout ce dont nous avons besoin. C'est ainsi que par exemple les dictionnaires en format papier sont de moins en moins consultés car il est plus pratique de chercher sur son téléphone la définition d'un mot, sa prononciation voire son emploi ou sa traduction.

Ce grand essor qu'ont connu les technologies de l'information touche essentiellement les jeunes générations : nos apprenants sont qualifiés de 'natifs numériques', de 'génération y', 'génération digitale', etc. Michel Serres (2002) appelle cette génération "petite poucette " car elle utilise fréquemment ses pouces pour clavarder et envoyer des SMS. L'utilisation intensive des mobiles et de l'internet dépasse chez ces jeunes un simple effet de mode ou de confort; le lien qu'ils ont développé avec les médias sociaux traduit une nouvelle façon d'être et de communiquer. Prensky (2001) va même jusqu'à opposer 'natifs numériques' aux 'migrants numériques'; selon lui, les premiers ont grandi dans un environnement technologique, ils sont imprégnés par les nouvelles technologies et leur culture est reliée au web, à internet, aux ordinateurs et aux jeux vidéo; quant aux seconds ils sont habitués au format papier et doivent se familiariser au numérique, notamment en utilisant les nouvelles technologies.

Pour Michel Serres, les anciennes générations vivaient dans un espace métrique qualifié par les distances et les concentrations des personnes, elles étudiaient au sein d'un collectif : 'l'école', 'l'amphithéâtre', 'la bibliothèque'... Les jeunes habitent aujourd'hui un espace virtuel, éclaté, "ils hantent un espace topologique de voisinages " (2002, p. 20), un étudiant peut suivre un cours en ligne à n'importe quel moment chez lui ou ailleurs, il peut le comparer à un autre, sélectionner ce dont il a besoin, établir des comparaisons et en faire ressortir les points forts ou les points faibles ! Mais face à ces grandes mutations, qu'en est-il du savoir-faire et du savoir-apprendre? Certes le savoir est là, partout, disponible, distribué sur la toile mais est-ce suffisant pour apprendre? Apprentissage en ligne et apprentissage en présentiel, est-il question seulement d'un changement de support et de cadre d'apprentissage ou faut-il prendre d'autres facteurs en considération pour suivre ces mutations technologiques? Comment la présence peut-elle rencontrer la distance et la redéfinir?

Dans cet article, nous souhaitons questionner cette effervescence dans son rapport à l'apprentissage. La génération 'petite poucette' apprend-elle plus rapidement en ligne? Quels cours à distance pour cette nouvelle génération? L'enseignant a-t-il la même présence dans un espace réel d'apprentissage que dans un espace virtuel ou est-il question d'adopter une nouvelle pédagogie d'apprentissage?

Nous nous sommes interrogées sur cette relation en partant d'une expérience d'enseignement hybride de la langue française que nous avons menée avec des étudiants en sciences, une expérience qui nous a permis de nous interroger sur notre posture d'enseignante. Le lecteur l'aura compris, répondre à ces questions revient à partager un ensemble de réflexions émanant principalement d'un changement qui va de la présence vers la distance. Nous présenterons, dans un premier temps, le contexte global dans lequel a été programmé cet enseignement hybride, ensuite nous exposerons les facteurs qu'on devrait prendre en considération dans son projet d'enseignement en ligne.

\section{Retour sur expérience}

La faculté des sciences-Meknès de l'université Moulay Ismaïl est un établissement à accès ouvert. Les effectifs des étudiants qui rejoignent les bancs des amphithéâtres sont de plus en plus pléthoriques. Pour l'année universitaire 2016-2017, la FSM a compté à peu près 3000 étudiants inscrits en 1 ère année. Le 
problème majeur que rencontrent actuellement les jeunes étudiants au Maroc est celui de la langue d'enseignement adoptée dans le cycle supérieur : étant donné qu'ils ont poursuivi leurs études des matières scientifiques, au secondaire, en langue arabe, arrivés à l'université ils ne sont pas en mesure de saisir les contenus des disciplines véhiculées en langue française. Cette transition linguistique constitue un handicap dont les conséquences affectent les profils et l'avenir des étudiants. Pour remédier à ce problème majeur, le Ministère de l'enseignement supérieur, de la recherche scientifique et de la formation des cadres a introduit l'enseignement du Français dans les universités depuis 2003. L'enseignement de la langue française fait donc partie de l'architecture des filières; nous dispensons ainsi des cours de Français sur objectif spécifique et sur objectif universitaire pour répondre aux besoins des étudiants par rapport aux exigences académiques des disciplines enseignées. Néanmoins, il faudrait signaler que pour des raisons logistiques, l'administration ne peut effectuer des tests de positionnement et par conséquent ne peut établir une répartition des groupes-classes en fonction des niveaux des étudiants. Ainsi le principal problème auquel est confronté le professeur de langue est l'hétérogénéité et la disparité des niveaux au sein du même groupe.

Dans ce contexte et devant ces contraintes, l'introduction de la formation en ligne présente plusieurs avantages. Elle permet de passer d'un cadre fermé d'apprentissage où le nombre des étudiants ne cesse de croître vers un cadre ouvert où tout un chacun peut travailler en autonomie selon son rythme et en fonction de ses possibilités. En outre, grâce à cet enseignement nous pouvons :

- proposer différents parcours pédagogiques contextualisés en migrant vers des dispositifs plus riches : la plateforme permet d'offrir des ressources et des activités adaptées aux publics issus de filières diverses;

- donner un accès privilégié à la formation des étudiants, surtout ceux qui sont en grande difficulté, en leur proposant des parcours individualisés;

- enrichir le volume de temps alloué à la matière;

- exploiter les potentiels des techniques numériques pour enrichir la formation : utilisation du multimédia, connexion avec d'autres plateformes, etc.

Néanmoins, il faut souligner qu'au sein de l'établissement, ce qui est mis à la disposition des professeurs, c'est la plateforme Moodle, mais sans aucun engagement pour y inscrire les étudiants. La surpopulation s'avère un obstacle majeur pour l'institution qui a opté pour un enseignement en amphithéâtre des matières transversales tels le Français et la communication. Assister les professeurs dans un enseignement à distance ne relève donc pas des priorités de l'établissement.

S'engager dans une aventure d'enseignement en ligne dans de telles conditions peut sembler une gageure : nombre d'étudiants, absence d'inscription sur la plateforme, absence de tuteurs, impossibilité d'effectuer une évaluation au cours de la formation, absentéisme, etc. Néanmoins, notre objectif était de ne pas céder au désespoir et d'aider dans la mesure du possible des étudiants qui négocient leur avenir au prix de l'apprentissage d'une langue étrangère. En 2016, nous avons scénarisé un dispositif hybride qui prévoyait des activités en présentiel et d'autres à distance tout en veillant à maintenir une cohésion en terme d'articulation entre les deux espaces pédagogiques. Nous avons effectué cette expérience avec 300 étudiants de la filière science de la vie, de la terre et de l'univers (SVTU). Ainsi, les thématiques étaient reliées aux disciplines des spécialités enseignées : écologie et environnement, énergies renouvelables et nanotechnologies. Les activités ciblaient essentiellement la compréhension et la production écrite et orale. L'objectif était de comprendre et d'analyser un document scientifique et de prendre des notes à partir d'un cours des dites disciplines. En parallèle, l'étudiant était amené à réfléchir de manière inductive sur les structures de la langue à travers des exercices interactifs. Le distanciel a permis à l'étudiant de préparer 
en amont le cours : travail en ligne de la compréhension et de la production écrite par le biais d'un ensemble de supports écrits ou audio-visuels. Les séances en présentiel étaient des séances de guidage, d'ancrage des acquis, de mise au point pour une meilleure maîtrise du scénario pédagogique et des objectifs fixés.

L'expérience a eu un grand succès auprès des étudiants non seulement de la filière SVTU mais aussi physique-chimie et sciences mathématiques. Les étudiants de ces filières ont commencé à assister aux cours en présentiel pour pouvoir suivre cet enseignement en ligne. Néanmoins dans ce contexte de la massification et pour les raisons précitées, nous n'avons pas pu mesurer l'évolution des acquis d'apprentissage des étudiants. Ainsi loin de toute perspective positiviste, le retour sur cette expérience n'a pas pour objectif de démontrer l'efficacité du produit développé, mais plutôt de comprendre et d'analyser la dynamique entre l'objet développé et la perception des acteurs (enseignant et apprenants) à travers les expériences d'usage.

\section{Réflexions}

L'analyse et l'évaluation de cette expérimentation a fait ressortir plusieurs questions importantes qui méritent d'être étudiées pour améliorer la qualité de cet enseignement en ligne.

\section{Première réflexion}

Notre première réflexion porte sur la fascination technologique. En effet les pratiques pédagogiques dans l'enseignement en ligne sont souvent modelées par des présupposés liés au rôle du numérique comme le schématise la figure suivante :

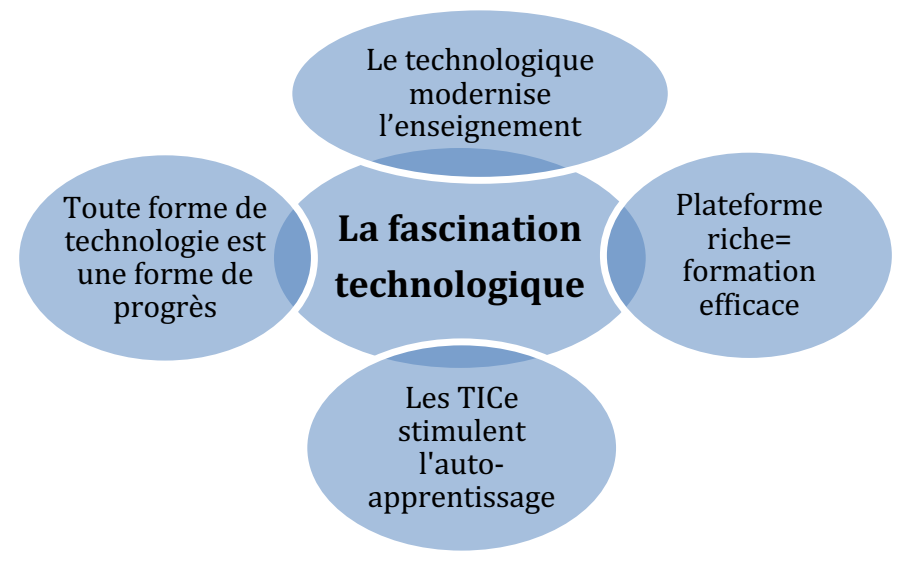

Figure 1. Des présupposés liés à la fascination technologique

Au début de notre première expérience, les Technologies de l'Information et de la Communication pour l'enseignement (TICE) étaient pour les collègues et nous-mêmes objet de toutes les promesses! Avec beaucoup d'enthousiasme et de motivation, ces cours ont été mis en ligne. La convivialité de l'interface, 
l'allègement et la miniaturisation des matériaux, la relative pérennité de l'information, l'interopérabilité des données entre la plateforme de l'université et d'autres applications en ligne étaient tous des aspects qui nous semblaient stimuler l'auto-apprentissage et gommer les traditionnelles barrières liées à l'enseignement formel. Un présupposé évident gérait la démarche du travail : plus la plateforme serait riche, plus la formation serait efficace. Autrement dit, pour permettre à nos étudiants adultes, donc majeurs et responsables, de dépasser leurs lacunes, nous pensions qu'il suffisait de mettre en ligne tout ce dont avaient besoin.

Cette fascination technologique avait aussi un effet sur les étudiants : surfeurs actifs et chevronnés sur le Net, au début ils étaient très motivés par cette nouvelle forme des cours. Mais au fil du semestre, nous avions remarqué que leur enthousiasme déclinait car ils avaient vite constaté que les cours en ligne nécessitaient un grand investissement qui n'avait rien à avoir avec les chats et les conversations sur les réseaux sociaux.

\section{Deuxième réflexion}

Notre deuxième réflexion s'inscrit dans la continuité de la précédente et porte sur la question de l'autonomie. Souvent nous présupposons que les étudiants habitués à surfer en ligne sont des étudiants autonomes. L'addiction des jeunes aux technologies numériques peut induire en erreur : 'génération numérique', 'génération y', 'génération digitale'... sont toutes des qualifications qui laissent croire que l'autonomie d'apprentissage est presque 'innée' ou acquise d'une façon quasiment naturelle chez nos apprenants. Partir de ce présupposé dans la conception et la mise en place des dispositifs en ligne écarterait plusieurs paramètres déterminants pour la réussite d'un autoapprentissage.

Porcher pense que « l'autonomie est toujours quelque chose vers quoi l'on va, que l'on construit, que l'on ne possède jamais totalement » (1981/1989, p. 61). II s'agit donc d'un apprentissage progressif, d'une marche, d'un cheminement qu'on trace vers l'autonomie. Il est question de guider et de baliser un parcours où l'autonomie est programmée comme une fin, comme une compétence à faire développer. Ainsi il ne faut pas partir d'un présupposé d'autonomie mais plutôt en supposer un déficit pour gérer autrement les pratiques pédagogiques programmées à distance.

Nous avons donc choisi quelques entrées pour développer l'autonomie de nos étudiants, et la motivation constituait un facteur principal. Nous sommes ainsi parties du principe que pour favoriser une dynamique motivationnelle, il faudrait concevoir avec les étudiants un programme centré sur leurs intérêts et leurs besoins. Le premier pas était, donc, de les impliquer dans l'élaboration des objectifs du dispositif hybride. Ainsi, nous avons diffusé un questionnaire pour délimiter leurs attentes et en fonction de leurs réponses nous avons fixé les objectifs du contenu : lexique scientifique relatif aux disciplines enseignées, étude et analyse des consignes des examens, et rédaction d'un résumé d'un cours. Ces objectifs étaient surtout reliés aux difficultés qu'ils rencontraient pour saisir les cours des spécialités ou pour répondre aux questions des examens. Avant le lancement de la formation, notre intention était de conduire les débats vers l'élaboration d'un contrat pédagogique. II s'agissait d'un contrat moral où l'apprenant était responsabilisé à pratiquer l'auto-apprentissage en dehors du cadre de la classe.

L'auto-apprentissage pour nous est loin d'être synonyme de liberté totale. Bien au contraire, nous pensons que pour installer une autonomie dans l'apprentissage en ligne et pour maintenir la motivation, il faudrait introduire la notion de 'contrainte' dans la conception du déroulement de la formation. La contrainte, dans ce cadre, n'est pas un équivalent de pression mais une expression de volonté partagée, objet de consensus entre apprenants et enseignant à réaliser un ensemble d'actions pour atteindre un objectif précis. Dans la mise en place du dispositif, ce contrat pédagogique a été régi par un 'carnet de bord' qui permet à l'étudiant de mesurer sa progression. Les rubriques prédéfinies du carnet de bord contribuent à une réflexion sur les pratiques, les difficultés rencontrées, les techniques utilisées et surtout le temps 
consacré à l'auto-apprentissage. De ce fait, cet outil ne permet pas seulement une autonomisation dans l'apprentissage, mais aussi une conscientisation de l'intérêt d'un travail intensif à distance. II s'agit d'une modalité programmée pour permettre le déconditionnement de nos apprenants habitués aux méthodes transmissives et dépendants du professeur et du cours en présentiel.

Au-delà de l'autonomie d'apprentissage et de l'engagement de l'étudiant dans le processus d'apprentissage, un autre facteur est à prendre en considération pour réussir son cours en ligne : il s'agit de la scénarisation.

\section{Des innovations qui imposent une nouvelle pédagogie}

Les cours en ligne nécessitent de nouvelles approches pédagogiques, différentes de celles qu'on applique en présentiel, car l'environnement d'apprentissage n'est plus le même. Une formation à distance se définit par opposition à la formation en présentiel, par la rupture de l'unité du temps et de l'espace et surtout par l'absence physique de l'enseignant (Peraya, 2011).

En présentiel, la présence physique de l'enseignant joue un rôle très important dans l'interaction. Depuis la maïeutique classique, plus précisément chez Socrate, l'enseignant est « un accoucheur » : le principe étant de ne pas énoncer soi-même la vérité mais permettre aux autres de la découvrir, c'est dans l'interaction et le questionnement que naît la connaissance. Le professeur est là pour guider, orienter, redresser les situations d'apprentissage et remettre les esprits sur la bonne voie de la connaissance. Mais, à distance, devant l'éclatement de l'unité de temps, de l'espace et de l'action, quelle maïeutique peut-on alors adopter pour apprendre à apprendre?

Mettre un cours magistral en ligne ou des documents en format PDF sur la plateforme ne relève pas de l'innovation pédagogique; il s'agit d'un retour vers les méthodes transmissives (appelées méthodes jésuites) où le premier souci de l'enseignant est de s'assurer que le discours est clairement transmis. Le véhicule technologique dans ce cas ne révolutionne pas l'apprentissage et n'implique pas une refonte des ressources, pas plus que "le camion qui amène les victuailles au supermarché n'améliore la nutrition d'une communauté » (Clark, 1983, p.101). Autrement dit, les étudiants peuvent surfer constamment sur ces contenus sans jamais les transformer en connaissances personnelles car sur le plan cognitif, le travail se limite à de la consultation.

Idéalement, la recherche dans le domaine de l'innovation pédagogique devrait s'appuyer sur le développement des théories pédagogiques pour construire un nouveau terrain d'apprentissage :

- le behaviorisme (modeler le comportement de l'apprenant à travers des situations où il est mis face à des stimulus),

- le cognitivisme et la méta-cognition (rendre l'apprenant conscient de la façon dont il construit ses connaissances),

- le constructuvisme (où la force de l'enseignant est de travailler sur l'erreur et de guider son apprenant sans aplanir les difficultés),

- le socio-constructivisme (où l'apprentissage est basé sur des situations d'échange et d'interaction).

Le développement de la pédagogie en ligne ne devrait pas écarter ces théories mais plutôt en tirer parti en les conjuguant au développement des outils technologiques pour améliorer la qualité de l'apprentissage. Le développement des plateformes, la mise à disposition de multiples outils pour l'expression et la communication nécessitent une appréhension plus profonde du rapport circulaire et systémique qu'elles génèrent entre pédagogie et technologie. 


\section{Conditions pour redéfinir la présence à distance}

Mettre un cours en ligne consiste à accompagner l'étudiant là où il se trouve. La plateforme est un espace où se rencontrent les outils techniques et les méthodes de formation pour devenir un moyen de construction de connaissances et de compétences. Cette construction devrait passer par des pédagogies actives, incitatives et interactives où il faut prévoir un rapport circulaire entre les outils technologiques et les objectifs pédagogiques, auxquels s'ajoutent les modalités d'évaluation et les compétences à acquérir. II s'agit d'un mécanisme qui ne fonctionne que si tous les éléments constituants sont bien articulés, comme l'illustre la figure 2 .

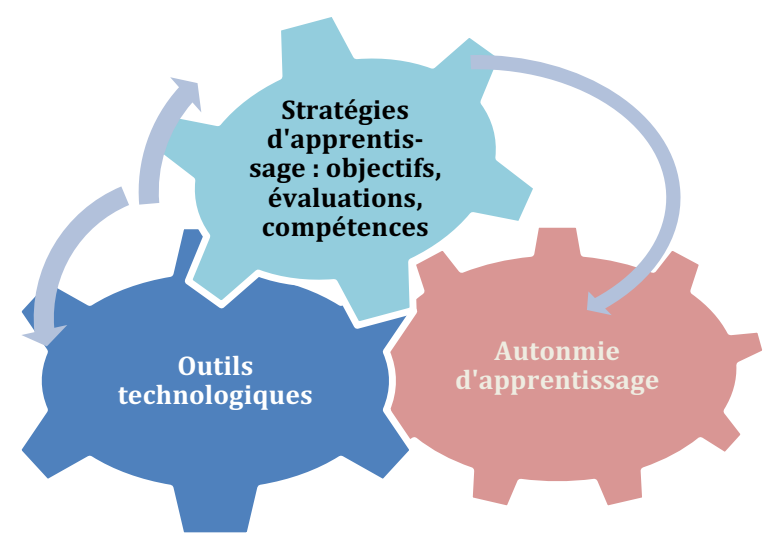

Figure 2. Le rapport systémique entre outils, stratégies et autonomie d'apprentissage

La première condition pour réaliser cette construction est la détermination des objectifs du projet pédagogique. Cette détermination touche l'intentionnalité qui inscrit les démarches d'apprentissage dans « une intention développementale. » (Tardif, 1994, p.5) Déterminer les objectifs pédagogiques d'un projet et l'intention de l'apprentissage en ligne, c'est inscrire la recherche de l'information dans une perspective constructiviste, dans la mesure où l'étudiant cible un champ de travail bien précis (Rabardel, 1995). Prenons à titre d'exemple un projet pédagogique sur l'argumentation avec un groupe d'étudiants de niveaux hétérogènes. Le professeur peut programmer pour tout le groupe une séquence commune " maîtriser les types d'arguments " mais il peut prévoir différentes activités selon des intentions qui correspondent aux besoins spécifiques de chaque étudiant : certains vont exprimer un jugement favorable ou défavorable, commenter un avis ou justifier de façon fine et détaillée; d'autres vont développer méthodiquement une argumentation en mettant en relief les points pertinents et structurer des propos pour convaincre. Le travail à distance permettrait à chaque étudiant de réaliser une tâche selon son projet d'apprentissage personnalisé.

La deuxième condition est reliée aux méthodes d'apprentissage et plus précisément à la scénarisation des cours. La construction d'un cours passe par des phases et des étapes qui doivent s'inscrire dans une logique de progression et d'interactivité. Nous proposons ici des phases' qui nous semblent nécessaires pour permettre à l'étudiant d'acquérir progressivement l'information en question, surtout si elles sont conjuguées aux théories de l'apprentissage citées plus haut.

\footnotetext{
${ }^{1}$ Ces phases ont été élaborées en collaboration avec les professeurs de l'Institut des Langues Vivantes de Liège dans le cadre d'un échange d'expérimentations.
} 
- Phase de sensibilisation : dans cette phase, l'enseignant introduit le thème général de son cours pour intéresser et motiver ses apprenants. On pourrait par exemple programmer une activité à réaliser pour éveiller leur curiosité (les approches béhavioristes peuvent être de mise).

- Phase d'exposition : dans cette phase, l'apprenant va être mis en contact avec le thème à exploiter pour atteindre les objectifs du cours (approches cognitivistes).

- Phase d'appropriation : c'est la phase de systématisation où des exercices de réflexions et d'application sont mis en œuvre pour permettre une bonne appropriation des connaissances.

- Phase d'assimilation : dans cette quatrième phase, l'apprenant doit pouvoir commencer à utiliser ses nouvelles connaissances tout en restant dans un cadre bien précis. C'est la phase de transition et de production.

- Phase de production : dans cette phase, l'enseignant proposera à l'apprenant de se lancer dans des constructions plus libres, seul ou en collaboration avec ses camarades en ligne à travers les plateformes collaboratives (approches socioconstructivistes). II pourra ainsi mettre à profit ses nouvelles connaissances et mesurer les effets positifs de son apprentissage.

A ces phases, on pourrait ajouter une phase d'auto-évaluation où le but n'est pas de cibler les erreurs de l'apprenant, mais de lui permettre de prendre conscience de ses progrès et éventuellement des opportunités qu'il a de s'améliorer. En fonction des objectifs du dispositif, certaines phases et certaines approches peuvent être plus développées que d'autres et leur réalisation devrait être essentiellement basée sur l'utilisation d'outils technologiques porteurs de potentiels. Autrement dit, il s'agit de choisir l'outil adéquat pour créer un contenu interactif.

Ceci nous permet d'aborder un troisième facteur relié à la conceptualisation des cours en ligne, il s'agit de « la littératie numérique ». Cette notion est fréquemment employée ces dernières années pour désigner la compétence de la maîtrise de l'utilisation de l'outil technologique. Une construction d'un cours en ligne exige que le professeur entre en interaction avec un ensemble diversifié d'outils technologiques pour pouvoir les déployer facilement dans des dispositifs d'apprentissage. Ainsi encourager l'innovation, c'est avant tout mettre à la disposition des enseignants de nouveaux outils et organiser des formations pour qu'ils puissent s'y familiariser. Sans leur disponibilité et leur maîtrise d'emploi, une intégration réussie des TICE ne serait possible. Souvent les enseignants qui refusent d'intégrer les TICE dans leurs cours trouvent qu'ils n'ont pas les compétences nécessaires et que la réalisation de ces cours est une activité chronophage. Le rôle de l'institution pour promouvoir l'innovation n'est pas d'investir uniquement dans l'achat du matériel mais de participer à la promotion de la pédagogie à travers la technologie. Ceci nous semble un préalable nécessaire pour cadrer les effets de la technologie sur la qualité de l'apprentissage, car ce rapport interactif entre les configurations techniques et le développement professionnel de l'enseignant a un impact sur la réalisation de dispositifs plus fins et mieux circonstanciés.

\section{Conclusion}

L'introduction du numérique dans l'enseignement à l'université a fait entrer la question de la pédagogie par la grande porte. Si le modèle expositif et transmissif (Viaud, 2015) continue à dominer dans l'enseignement supérieur en présentiel, à distance le professeur se rend compte facilement que cette approche ne permet pas d'atteindre les objectifs escomptés : de nouvelles approches s'imposent donc pour favoriser l'apprentissage. Certains éléments peuvent leurrer ceux qui tentent leurs premières expériences dans le domaine; par exemple la question de la fascination technologique ou de l'autonomie peuvent constituer un principal point de blocage. D'autres éléments comme la question de la scénarisation, 
le choix de l'outil technologique ou la motivation de l'étudiant, peuvent aider à gagner le pari. Tels les maillons d'une chaîne, ces éléments sont étroitement liés et réussir un cours en ligne dépend d'une prise en considération de leur articulation.

La transformation du rapport au savoir à travers le numérique impose de nouvelles modalités d'enseignement, et contrairement à ce qu'on l'on pourrait croire, la préparation d'un cours en ligne est un travail qui demande énergie et sueur dans la mesure où rien ne devrait être laissé au hasard, où toutes les étapes devraient être 'calculées' pour une atteinte efficace des objectifs prescrits en amont.

\section{Liste de références}

Clark, R. E. (1983). Reconsidering research on learning from media. Review of Educational Research. 17(2), p. 92-101.

Pereya, D. (2011). Un regard sur la « distance, vue de la «présence ». Dans Distances et savoirs, 2011(9), 445-452.

Porcher, L. (1989). Les chemins de la liberté. Dans B. André, (dir.), Autonomie et enseignement apprentissage des langues étrangères. Paris, Didier/Hatier (Ouvrage original publié en 1981).

Prensky, M. (2001). Digital natives, digital immigrants. Dans The Horizon, 9(5).

Rabardel, P. (1995). Les Hommes et les technologies, approche cognitive des instruments contemporains. Paris : Armand Colin.

Serres, M. (2012). Petite Poucette. Paris : Le pommier.

Tardif, J. (1996). Une condition incontournable aux promesses des NTIC en apprentissage : une pédagogie rigoureuse. Repéré sur le site d'OCCE de la Drôme à http://www.acgrenoble.fr/occe26/printemps/tardif/pedagogie.htm

Viaud, M. (2015). Les innovateurs silencieux. Histoire des pratiques d'enseignement à l'université depuis 1950. Grenoble : PUG. 